\title{
Controlling chondrocyte senescence
}

New research published in Science Translational Medicine implicates microRNA-204 in the mechanism by which oxidative stress results in altered chondrocyte metabolism and cartilage loss in osteoarthritis (OA).

"The molecular pathways governing the senescent phenotypes of chondrocytes in OA are not well-characterized," says Jin-Hong Kim, corresponding author of the new study.

The researchers first began studying miR-204 after pulling it from a small RNA sequencing screen of primary mouse chondrocytes that had been cultured in hyperoxic conditions to induce oxidative stress. They subsequently found that miR-204 is highly expressed in cartilage from patients with OA, specifically in parts of the cartilage that are most damaged.

By treating chondrocytes in vitro with the miRNA, the researchers showed that a major effect of miR-204 is to change chondrocyte activity and metabolism. miR-204 made these cells produce less extracellular matrix (ECM) and this ECM had a low proteoglycan content, similar to chondrocytes in OA cartilage.

Furthermore, intra-articular injection of miR-204 resulted in loss of proteoglycans and in cartilage damage and exacerbated cartilage loss in mice after destabilization of the medial meniscus (DMM; as a model of OA).

The researchers think that by reducing proteoglycan biosynthesis, the cartilage becomes less able to cope with load bearing.

Notably, intra-articular injection of an anti-miR oligonucleotide against miR-204 into DMM mice resulted in recovery of chondrocyte proteoglycan synthesis. These mice were able to put more weight on the affected leg than mice injected with control anti-miR oligonucleotides, indicating a reduction in pain.

"However, to treat chronic diseases such as OA in this

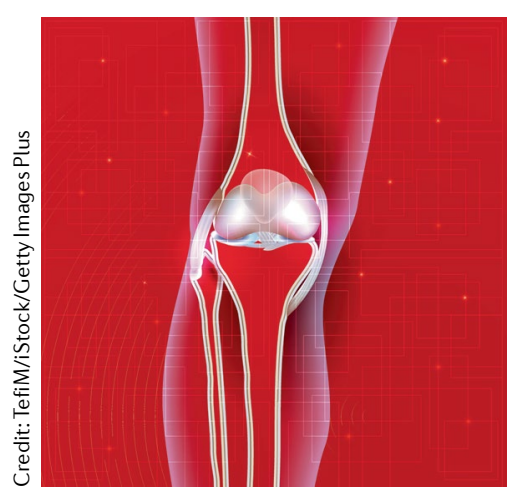

manner would require repeated dosing of anti-miR oligonucleotides as they are not particularly stable," cautions Kim. "So we are now attempting to develop protective vehicles or RNA modification methods to come up with a new therapeutic strategy to maximize the injection interval. Furthermore, we are designing RNA-based therapeutics using chondrocyte-affinity peptides for targeted intra-articular delivery."

Nicholas J. Bernard patients with $\mathrm{OA}$

ORIGINAL ARTICLE Kang, D. et al. Stressactivated miR-204 governs senescent phenotypes of chondrocytes to promote osteoarthritis development. Sci. TranslMed. 11, pii: eaar6659 (2019)

\section{Soluble CD83: a proresolving mediator in inflammatory arthritis?}

The soluble form of CD83 (sCD83) has immunomodulatory properties, including the ability to induce tolerogenic dendritic cell and regulatory $\mathrm{T}\left(\mathrm{T}_{\text {reg }}\right)$ cell responses, but its involvement in inflammatory arthritis has, until now, been unclear. "We show for the first time that SCD83 has anti-inflammatory properties in arthritis and induces resolution of inflammation," explains Elisabeth Zinser, co-corresponding author of a new study.

Given that dendritic cell activation and persistent stimulation of $T$ cells are important contributors to the pathogenesis of rheumatoid arthritis, the researchers sought to investigate the modulatory function of sCD83 in two mouse models of arthritis: antigen-induced arthritis (AIA) and $\mathrm{K} / \mathrm{B} \times \mathrm{N}$ serum transfer-induced arthritis.

"Interestingly, sCD83 treatment not only reduced joint swellings, but also inhibited expression of important pro-inflammatory cytokines," reports co-corresponding author Alexander Steinkasserer. In the AIA model, administration of $\mathrm{sCD} 83$ accelerated the resolution of inflammation compared with administration of saline. sCD83 also inhibited the expression of receptor activator of nuclear factor- $\mathrm{\kappa B}$ ligand (RANKL), a regulator of osteoclast differentiation, potentially explaining the $\mathrm{SCD} 83$-mediated reduction in osteoclast formation and attenuation of bone and cartilage destruction.

The proresolving effect of sCD83 was completely abrogated with an indoleamine 2,3-dioxygenase (IDO) inhibitor and partially abrogated with an anti-transforming growth factor- $\beta$ (TGF $\beta$ ) antibody.

IDO is an important modulator of T cell responses, functioning as an enzyme to convert tryptophan (an essential amino acid for T cell proliferation) into kynurenine (an inducer

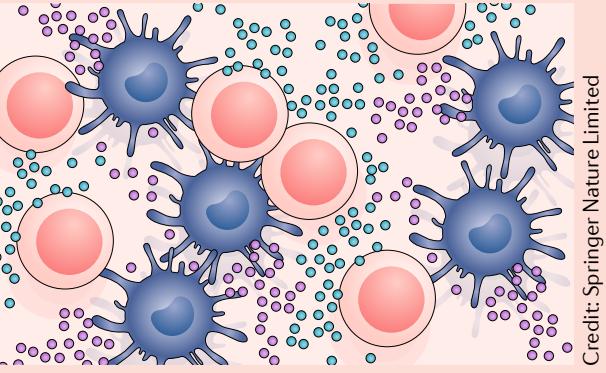

of $\mathrm{T}_{\text {reg }}$ cell responses), and as a signalling protein to induce TGF $\beta$ expression (an important cytokine for $\mathrm{T}_{\text {reg }}$ cell activity). Notably, treatment with the IDO inhibitor prevented the SCD83-induced accumulation of $\mathrm{T}_{\text {reg }}$ cells.

The researchers hypothesize that $\mathrm{SCD} 83$ functions through IDO to promote the expansion of $\mathrm{T}_{\text {reg }}$ cells, which induces the resolution of inflammation and inhibits osteoclast formation. "The next steps will

...administration of [soluble CD83] accelerated the resolution of inflammation be to further elucidate the underlying pro-resolving mechanisms induced by the sCD83-IDO axis," explains Steinkasserer. Jessica McHugh

ORIGINAL ARTICLE Royzman, D. et al. Soluble CD83 triggers resolution of arthritis and sustained inflammation control in IDO dependent manner. Front. Immunol. https://doi.org/10.3389/fimmu. 2019.00633 (2019) 